\title{
Microscopic modelling of perpendicular electronic transport in doped multiple quantum wells
}

\author{
Andreas Wacker and Antti-Pekka Jauho \\ Mikroelektronik Centret, Danmarks Tekniske Universitet, DK-2800 Lyngby \\ (to be published in Physica Scripta, Proceedings of the $17^{\text {th }}$ Nordic Semiconductor Meeting,
}

Trondheim, June 1996)

\begin{abstract}
We present a microscopic calculation of transport in strongly doped superlattices where domain formation is likely to occur. Our theoretical method is based on a current formula involving the spectral functions of the system, and thus allows, in principle, a systematic investigation of various interaction mechanisms. Taking into account impurity scattering and optical phonons we obtain a good quantitative agreement with existing experimental data from Helgesen and Finstad (J. Appl. Phys. 69, 2689, (1991)). Furthermore the calculated spectral functions indicate a significant increase of the average intersubband spacing compared to the bare level differences which might explain the experimental trend.
\end{abstract}

\section{INTRODUCTION}

In semiconductor superlattices the electric transport is dominated by resonances between the localized energy levels inside the wells. A resonance structure appears in the currentvoltage characteristics if the energy levels of different wells align [1] 3]. Between these resonances regions with negative differential conductivity (NDC) are likely to appear. This yields interesting current-voltage characteristics as already shown by Esaki and Chang [2]. If the voltage applied along the growth direction of the superlattice yields an average field which is in the NDC region, the homogeneous field distribution breaks up and electric field domains form causing many current branches in the current-voltage characteristic (almost equal to the number of quantum wells). This has been extensively studied experimentally during the last decade [4 9 .

Theoretically the complicated measured $I(U)$ characteristics could be reproduced using models based on the combination of rate equations between the wells and Poisson's equation [10 [12]. These models used simplified expressions for the tunnelling current between the wells [10], or a fitted local current-field characteristics [11]. A theoretical approach for the transport in superlattices with strong coupling between the wells has been reported in Ref. [13] with the restriction to one miniband. This restriction is not appropriate for situations where high field domains occur, as they are close to the resonance between the first and the second subband [9]. Nevertheless, no quantitative calculations have been performed so far. 
The aforementioned theories for domain formation showed that the electric field as well as the carrier density are almost constant within the field domains. Thus the current density is determined by intrinsic features of the superlattice. This is a much simpler situation than in many other semiconductor structures, such as the double-barrier resonant-tunnelling diode, where the contacts strongly influence the field profile and the current densities by carrier injection. Furthermore the single branches extend up to a current density, where the low field domain reaches the maximum of the current-field relation. Therefore the current maxima of the branches are a good estimate for the maximum in the current-field relation, which does not depend strongly on the contacts. The situation becomes slightly more complicated, if the superlattice exhibits fluctuation in its parameters, which have a strong impact impact on the individual branches [14].

In this paper we develop a unified microscopic theory, without adjustable parameters, to describe the underlying current-field relation. In contrast to the situation considered in Ref. [13], we consider the situation of weakly coupled quantum wells $\left(H_{1} \ll \Gamma\right)$ where $H_{1}$ is the coupling between the wells and $\Gamma$ is the broadening of the levels within the single wells. This means that the intrawell scattering rate is larger than the tunnelling rate. This is relevant to most experiments including that described in Ref. [6] with which we will compare our results.

\section{THE MODEL}

We consider weakly coupled semiconductor quantum wells of period $d$. Then the electrons are localized in the wells and a reasonable basis set of wave functions is given by a product of Wannier functions $\Psi^{\nu}(z-n d)$ localized in well $n$, and plane waves $e^{i \underline{k} \cdot \underline{r}}$. Here the $z$ direction is defined to be the growth direction and $\underline{k}, \underline{r}$ are vectors within the $(x, y)$ plane. $\nu$ denotes the subband within the well.

Restricting ourselves to the lowest two minibands (denoted by $a$ and $b$ ) and coupling between neighbouring wells we consider the following hamiltonian ( $F$ is the electric field, and $e<0$ is the charge of the electron):

$$
\begin{aligned}
& \hat{H}_{0}=\sum_{n, \underline{k}}\left[E_{n}^{a}(\underline{k}) a_{n}^{\dagger}(\underline{k}) a_{n}(\underline{k})+E_{n}^{b}(\underline{k}) b_{n}^{\dagger}(\underline{k}) b_{n}(\underline{k})\right] \\
& \hat{H}_{1}=\sum_{n, \underline{k}}\left[T_{1}^{a} a_{n+1}^{\dagger}(\underline{k}) a_{n}(\underline{k})+T_{1}^{b} b_{n+1}^{+}(\underline{k}) b_{n}(\underline{k})\right. \\
& \left.-e F R_{1}^{a b} a_{n+1}^{\dagger}(\underline{k}) b_{n}(\underline{k})-e F R_{1}^{b a} b_{n+1}^{\dagger}(\underline{k}) a_{n}(\underline{k})+h . c .\right] \\
& \hat{H}_{2}=\sum_{n, \underline{k}}\left[-e F\left(R_{0}^{a b} a_{n}^{\dagger}(\underline{k}) b_{n}(\underline{k})+R_{0}^{b a} b_{n}^{\dagger}(\underline{k}) a_{n}(\underline{k})\right)\right]
\end{aligned}
$$

with $E_{n}^{\nu}(\underline{k})=E^{\nu}+\hbar^{2} k^{2} /\left(2 m_{w}\right)-e F n\left(m_{w}\right.$ is the effective mass in the well), and the couplings $R_{h}^{\nu^{\prime} \nu}=\int d z \Psi^{\nu^{\prime}}(z-h d) z \Psi^{\nu}(z) .4\left|T_{1}^{a}\right|$ and $4\left|T_{1}^{b}\right|$ are the miniband widths of subband $a$ and $b$, respectively. The term $\hat{H}_{2}$ can be incorporated into the one electron states by diagonalizing $\hat{H}_{0}+\hat{H}_{2}$ [1]. This leads to renormalized coefficients in $\hat{H}_{0}$ and $\hat{H}_{1}$ but does not change the structure of the problem for a homogeneous electric field. In a similar way higher subbands and continuum states will change the coefficients, so we prefer to omit $\hat{H}_{2}$ in the following. 
The total current from subband $\nu$ in well $n$ to subband $\mu$ in well $n+1$ can be described by the following expression which is derived in Sect. 9.3 of Ref. [15]:

$$
\begin{aligned}
& J_{n \rightarrow n+1}^{\nu \rightarrow \mu}=2 e \sum_{\underline{k}^{\prime}, \underline{k}}\left|H_{(n+1) \underline{k}, n \underline{k^{\prime}}}^{\mu, \nu}\right|^{2} \int_{-\infty}^{\infty} \frac{d \mathcal{E}}{2 \pi \hbar} A_{n}^{\nu}\left(\underline{k^{\prime}}, \mathcal{E}\right) \\
& \cdot A_{n+1}^{\mu}\left(\underline{k}, \mathcal{E}+\mu_{n}-\mu_{n+1}\right)\left[n_{F}(\mathcal{E})-n_{F}\left(\mathcal{E}+\mu_{n}-\mu_{n+1}\right)\right] .
\end{aligned}
$$

Here $\mu_{n}$ is the electro-chemical potential in well $n$ and $n_{F}(\mathcal{E})=\left(1+e^{\beta \mathcal{E}}\right)^{-1}$ is the Fermi function. The energy $\mathcal{E}$ is measured with respect to $\mu_{n}$. Note that for equal densities in both quantum wells we have $\mu_{n}-\mu_{n+1}=e F d$.

$A_{n}^{\nu}(\underline{k}, \mathcal{E})$ denotes the spectral function for the state $\underline{k}$ of the subband $\nu$ in well number $n$. It is calculated in equilibrium neglecting the coupling to the other wells and is related to the retarded self-energy $\Sigma_{n}^{\nu \operatorname{ret}}(\underline{k}, \mathcal{E})$ via

$$
A_{n}^{\nu}(\underline{k}, \mathcal{E})=\frac{-2 \operatorname{Im} \Sigma_{n}^{\nu \text { ret }}}{\left(\mathcal{E}+\mu_{n}-E_{n}^{\nu}(\underline{k})-\operatorname{Re} \Sigma_{n}^{\nu \text { ret }}\right)^{2}+\left(\operatorname{Im} \Sigma_{n}^{\nu \text { ret }}\right)^{2}}
$$

If no perturbation is present the state $\underline{k}$ has a fixed energy, $E_{n}^{\nu}(\underline{k})$, and the spectral function becomes a $\delta$-function $A_{n}^{\nu}(\underline{k}, \mathcal{E})=2 \pi \delta\left(\mathcal{E}+\mu_{n}-E_{n}^{\nu}(\underline{k})\right)$. If scattering is present the states $\underline{k}$ are no longer eigenstates of the full hamiltonian and the spectral function becomes smeared out, which is often modeled by a Lorentzian using a constant $\Sigma_{n}^{\nu \text { ret }}$.

While the full derivation is slightly tedious [15] the formula (41) can be motivated quite easily: In the long-time limit energy has to be conserved during transitions caused by the time-independent interwell couplings $H_{(n+1) \underline{k}, n \underline{k}^{\prime}}^{\mu, \nu}$ Therefore we have to consider tunnelling processes for a certain energy $\mathcal{E}$ and integrate over $\mathcal{E}$ afterwards. The factor $\left[n_{F}(\mathcal{E})-n_{F}\left(\mathcal{E}+\mu_{n}-\mu_{n+1}\right)\right]$ takes into account the thermal occupation at the given energy in both wells. The free particle state $\underline{k}^{\prime}$ has a weight $A_{n}^{\nu}\left(\underline{k}^{\prime}, \mathcal{E}\right) /(2 \pi)$ in well $n$. Its transition probability to the state $\underline{k}$ in well $n+1$ is given by $2 \pi\left|H_{(n+1) \underline{k}, n \underline{k}^{\prime}}^{\mu, \nu}\right|^{2} / \hbar$. The final state has a weight $A_{n+1}^{\mu}\left(\underline{k}, \mathcal{E}+\mu_{n}-\mu_{n+1}\right) /(2 \pi)$ at the given energy. Obviously one has to sum over all free particle states $\underline{k}, \underline{k}^{\prime}$. Finally, the factor 2 is due to the spin degeneracy.

We calculate the self-energy $\Sigma^{a \operatorname{ret}}(\underline{k}, \mathcal{E})$ for the lower subband for impurity scattering against the ionized donors within the self-consistent single-site-approximation (SSA) (which contains all noncrossing diagrams as shown in the inset of Fig. 1). This approximation was also used in Ref. [16] to calculate the spectral functions in a single quantum well. The matrix element $V_{h}(\underline{p})$ of the interaction is calculated from the given Wannier functions both for impurities in the same well $(h=0)$ and for remote impurities located in the $h$ th well counted from the electron, which become important for $p d h<1$. We consider screening by the free 2D electron gas located both in the same well and in different wells within the $T=0$ Random Phase Approximation. The screened matrix element for impurity scattering is denoted by $V_{h}^{\text {sc }}(\underline{p})$. Within the Born approximation the total scattering rate with momentum transfer $\underline{p}$ is proportional to $\sum_{h}\left|V_{h}^{\mathrm{sc}}(\underline{p})\right|^{2}$. In Fig. 四 we have plotted this quantity as well as $\left|V_{0}^{\mathrm{sc}}(\underline{p})\right|^{2}$ as a function of $p$. For comparison we have also shown the result for the case when screening and scattering is restricted within the same well; this is larger than $\left|V_{0}^{\mathrm{sc}}(\underline{p})\right|^{2}$ because of the weaker screening. From Fig. 11 we can see that even for 
$p=0$ the main contribution to the total scattering $\sum_{h}\left|V_{h}^{\mathrm{sc}}(\underline{p})\right|^{2}$ comes from the scattering within the well $\left|V_{0}^{\mathrm{sc}}(\underline{p})\right|^{2}$. Thus we can restrict ourselves to this contribution in our case.

The self-consistent Born-Approximation (BA) (consisting of the first diagram shown in the inset of Fig. 1) breaks down for matrix elements $\pi c V \approx 1$, where $c=m_{w} /\left(2 \pi \hbar^{2}\right)$ is the density of states per spin of the free 2D electron gas. Fig. 1 tells us that we are in the range where the higher order diagrams become important which invokes the need for the SSA.

For the calculation of the self-energy $\Sigma^{b \operatorname{ret}}(\underline{k}, \mathcal{E})$ of the upper subband we additionally include the emission of optical phonons for scattering between the upper to the lower subband describing the intersubband relaxation. This leads to an additional imaginary part $-\Gamma_{\mathrm{ph}}^{b}$ in the self-energy $\Sigma^{b \text { ret }}(\underline{k}, \mathcal{E})$.

\section{RESULTS OF THE CALCULATION}

Here we apply our approach to the experimental situation of Refs. [6, 17]. The $\mathrm{Al}_{0.3} \mathrm{Ga}_{0.7} \mathrm{As}$ superlattice used there had a nominal barrier width $b=12 \mathrm{~nm}$ and a well width $w=8 \mathrm{~nm}$. The middle $7 \mathrm{~nm}$ of the wells were n-doped with a doping density $N_{D} / A=8.75 \cdot 10^{15} / \mathrm{m}^{2}$. We calculate the coefficients in Eqs. (1, 2, 3) within the KronigPenney model assuming a parabolic dispersion with the conduction band offset $\Delta E_{c}=0.24$ $\mathrm{eV}$, and the effectives masses $m_{w}=0.067 m_{e}$ and $m_{b}=0.0919 m_{e}$ for well and barrier, respectively [18]. The resulting parameters are given in Table [1. Just as in the more sophisticated calculation used in Ref. [6,17] the calculated subband spacing $E_{b}-E_{a}=117.9 \mathrm{meV}$ is smaller than the experimental value $124 \mathrm{meV}$ determined by intersubband absorption.

We approximated the doping profile by two $\delta$-doping layers located at a distance of $1.75 \mathrm{~nm}$ from the middle of the well. The calculated spectral functions for subband $a$ are given in Fig. 2 for both the BA and the SSA. For $E_{k}=0.02 \mathrm{eV}$ both spectral functions $A^{a}\left(E_{k}, E_{s}\right)$ (where $E_{s}=\mathcal{E}+\mu-E_{a}$ is measured with respect to $E_{a}$ ) are quite similar and have approximately a Lorentzian shape with a half width of $\Gamma^{a} \approx 0.01 \mathrm{eV}$. In contrast to this for $E_{k}=0$ the spectral function exhibit significant differences for the BA and the SSA. Within both approximations they exhibit a sharp onset at $E_{s} \approx-0.02 \mathrm{eV}$ and are significantly different from Lorentzians.

The broad width of the spectral functions has obviously an impact for the optical absorption $\alpha(\omega)$ between the subbands which is proportional to

$$
\alpha(\omega) \propto \sum_{\underline{k}} \int_{-\infty}^{\infty} d \mathcal{E} n_{F}(\mathcal{E}) A^{a}(\underline{k}, \mathcal{E}) A^{b}(\underline{k}, \mathcal{E}-\hbar \omega) .
$$

Our calculation reveals a maximum at $\hbar \omega=123.5 \mathrm{meV}$ which is in excellent agreement with the experimental value. Nevertheless the full width at half maximum (35 meV) is larger than the experimental value $19 \mathrm{meV}$. (Within the $\mathrm{BA}$ the maximum is at $\hbar \omega=123 \mathrm{meV}$ and the width is $30 \mathrm{meV}$.) This shows that the measured interssubband spacing may deviate significantly form the bare energy levels for strongly doped samples.

For the given density of carriers provided by the doping we have calculated the chemical potential for the actual density of states determined from the spectral functions.

Finally we compute the current from Eq. (4). We use zero temperature and the Fermi functions $n_{F}(\mathcal{E})$ become step functions $\Theta(-\mathcal{E})$. Then only the lowest subband is occupied 
in equilibrium and the total current is given by the sum $I=I^{a \rightarrow a}+I^{a \rightarrow b}$. Using the nominal sample parameters we obtain current maxima for the current-field characteristics which are almost an order of magnitude lower than the currents measured in the respective regions. This indicates that the coupling between the wells should be stronger. Using the barrier width $b=10.5 \mathrm{~nm}$ we find good agreement with the experimental data. In their own theoretical analysis of the data Helgesen, Finstad, and Johannessen [17] use the width $b=10.8 \mathrm{~nm}$, which indicates that they consider this value to be within the experimental uncertainty. The result of the calculation for $b=10.5$ is given in Fig. 3. The $I(e F d)$ relation exhibits a first maximum at $e F_{M} d=13 \mathrm{meV}$ with a maximum current of $I_{M}=0.592 \mathrm{~mA}$ and a minimum at $e F_{m} d=69 \mathrm{meV}$ with a current $I_{m}=0.086 \mathrm{~mA}$. At $e F d=123 \mathrm{meV}$ there is a second maximum due to the resonance between the first and the second subband with a maximum current $I=29.4 \mathrm{~mA}$. The presence of a range with negative differential conductivity for $13 \mathrm{meV}<e F d<69 \mathrm{meV}$ causes an instability leading to the formation of field domains which is discussed in detail in Ref. [19 for an arbitrary $v(F)$ relation. The domain branches in the characteristic should exhibit a maximum current $I_{M}$ if the high field domain is located at the anode and a lower current if the high field domain is located at the cathode, which concerns the experimental situation here [6]. We obtain very similar results for the currents within the BA.

Let us compare these data with the experimental results. In Fig. 2 of Ref. [6] one can identify the first resonant maximum very well before the domain formation sets in. It is located at $U=0.3 \mathrm{~V}, I=0.64 \mathrm{~mA}$. Dividing the voltage by the number of periods $N=23$ we obtain $e F d=13 \mathrm{meV}$. Thus we have excellent agreement of both the position and the height of the peak. The low field resistance for the experimental $I(V)$ curves is $R \approx 570 \Omega$ for the 35 well superlattice (Fig. 1 of Ref. [17]) and $R \approx 331 \Omega$ for the 23 well superlattice (Fig. 2 of Ref. [6]). Dividing by the number of wells we obtain $d(e F d) / d I=16 \Omega$ and $d(e F d) / d I=14 \Omega$, respectively, which are in good agreement with our calculated value $d(e F d) / d I=12 \Omega$. The domain branches found experimentally exhibit maximum currents in the range between $0.35 \mathrm{~mA}$ and $0.55 \mathrm{~mA}$ which are lower than $I_{M}$ as common for field profiles where the high field domain located at the cathode.

In Fig. 3 we have also plotted some data points taken from Fig. 1 of Ref. [17] (we divide the experimental voltage by the number of periods to obtain the effective field $e F d$ ). We can see that the onset of the second peak occurs for larger effective fields experimentally than in our calculation. This might be related to the fact, that a part of the voltage may drop outside the superlattice, so that the electric field in the sample is smaller than the estimation $U / N d$ used. Good agreement is found if we assume that a voltage drop $U_{c}=0.33 \mathrm{~V}$ occurs in the contact.

In Fig. 3 of Ref. [17 the experimental current is given in terms of $\epsilon=1.24 \mathrm{meV}-U / N$. The authors do not state that they found a maximum at $U / N=1.24 \mathrm{meV}$ but just take this value from the measured intersubband spacing. Taking the voltage drop in the contact into account we have to use the value $\epsilon=124 \mathrm{meV}-U_{c} / N-e F d$ for our theoretical data. Then we obtain good agreement between the experimental data and the calculated curve as shown in Fig. 4 .

Analogously to Ref. [10] we calculated the current-voltage characteristic with domain formation using Eq. 4 for the calculation of the current from one well to the next and the discretized Poisson equation. Here we assumed fast relaxation within the wells, so that the 
upper subband is not occupied. Like in the experiment [6] we consider a 23 well superlattice. For the electron density $n_{i}$ in well $i$ we apply the boundary conditions $n_{0}=n_{23}=0.95 N_{D} / A$. The resulting current-voltage characteristic for sweep up is shown in Fig. 5 which is in good agreement with the experimental findings. The high field domain is located at the anode in our simulation which is typical for the boundary conditions used and for sufficiently large dopings as can easily be understood within the general theory given in Ref. [19].

\section{CONCLUSION}

We have developed a microscopic model to calculate the current in coupled quantum wells. The self-energies, which give the renormalized energies and widths of the spectral functions, are calculated directly from the nominal parameters describing the superlattice, without invoking additional adjustable parameters. The results for the current are in good quantitative agreement with the data from Ref. [6] if we assume the barrier width to be $1.5 \mathrm{~nm}$ less than the nominal value. We find good agreement for the low field behaviour including the position and height of the first maximum. Regarding the second peak the shape of the resonance is in good agreement if we assume, that $0.33 \mathrm{~V}$ of the total voltage drop outside the superlattice. Furthermore we have shown that the actual shape of the spectral functions due to the scattering seems to be responsible for a significant shift in the maximum of intersubband absorption.

\section{ACKNOWLEDGEMENT}

We want to thank B. Hu and K. Johnsen for helpful discussions. One of us (A.W.) gratefully acknowledges financial support from the German science foundation DFG. 


\section{REFERENCES}

11] Kazarinov, R. F. and Suris, R. A., Sov. Phys. Semicond. 6, 120 (1972).

[2] Esaki, L. and Chang, L. L., Phys. Rev. Lett. 33, 495 (1974).

[3] Capasso, F., Mohammed, K., and Cho, A. Y., Appl. Phys. Lett. 48, 478 (1986).

[4] Kawamura, Y., Wakita, K., Asahi, H., and Kurumada, K., Jpn. J. Appl. Phys. 25, L928 (1986).

[5] Choi, K. K. et al., Phys. Rev. B 35, 4172 (1987).

[6] Helgesen, P. and Finstad, T. G., in Proceedings of the $14^{\text {th }}$ Nordic Semiconductor Meeting, edited by Hansen, O. (University of Århus, 1990), p. 323.

[7] Grahn, H. T., Haug, R. J., Müller, W., and Ploog, K., Phys. Rev. Lett. 67, 1618 (1991).

[8] Kastrup, J. et al., Appl. Phys. Lett. 65, 1808 (1994).

[9] Kwok, S. H. et al., Phys. Rev. B 51, 9943 (1995).

[10] Prengel, F., Wacker, A., and Schöll, E., Phys. Rev. B 50, 1705 (1994).

[11] Bonilla, L. L. et al., Phys. Rev. B 50, 8644 (1994).

[12] Miller, D. and Laikhtman, B., Phys. Rev. B 50, 18426 (1994).

[13] Laikhtman, B. and Miller, D., Phys. Rev. B 48, 5395 (1993).

[14] Wacker, A. et al., Phys. Rev. B 52, 13788 (1995).

[15] Mahan, G. D., Many-Particle Physics (Plenum, New York, 1990).

[16] Serre, J., Ghazali, A., and Gold, A., Phys. Rev. B 39, 8499 (1989).

[17] Helgesen, P., Finstad, T. G., and Johannessen, K., J. Appl. Phys. 69, 2689 (1991).

[18] Properties of Aluminium Gallium Arsenide, edited by Adachi, S. (INSPEC, London, 1993).

[19] Wacker, A., Moscoso, M., Kindelan, M., and Bonilla, L. L., (1996), submitted to Phys. Rev. B. 


\section{FIGURES}

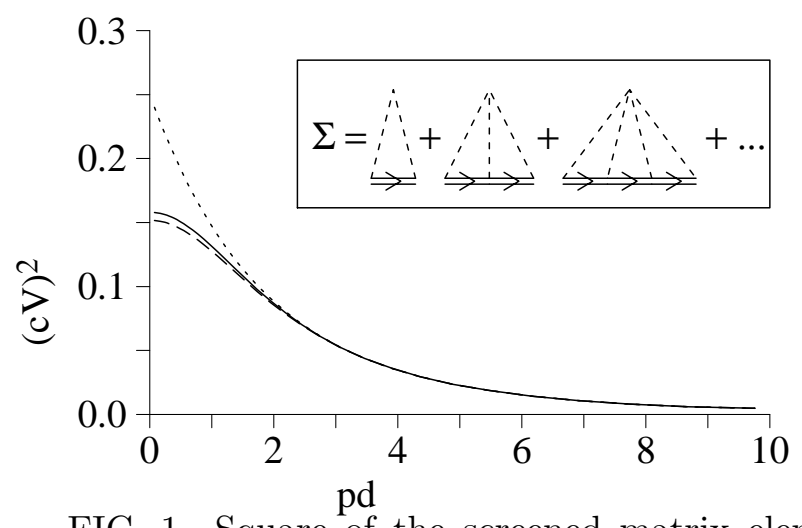

FIG. 1. Square of the screened matrix element for impurity scattering. The full line gives $\sum_{h}\left|V_{h}^{\mathrm{sc}}(\underline{p})\right|^{2}$ which determines the scattering in the BA. The dashed line gives $\left|V_{0}^{\mathrm{sc}}(\underline{p})\right|^{2}$. The dotted line gives the same expression within the restriction to screening by electrons from the same well. The inset depicts the diagrams contained in the SSA.

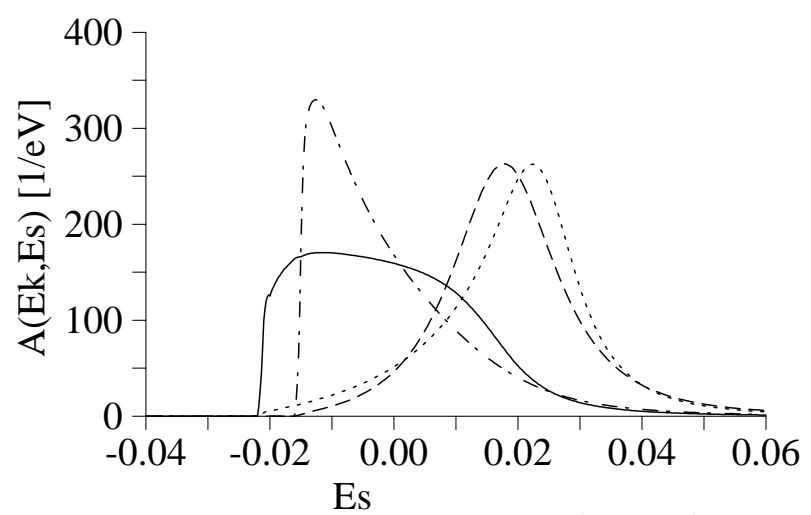

FIG. 2. Spectral functions $A^{a}\left(E_{k}, E_{s}\right)$ for the first subband. The full and dotted line give the result within the SSA for $E_{k}=0$ and $E_{k}=0.02 \mathrm{eV}$, respectively. The dashed-dotted and dashed line give the result within the BA for $E_{k}=0$ and $E_{k}=0.02 \mathrm{eV}$, respectively. The Energy $E_{s}$ is measured with respect to the bottom of the first subband.

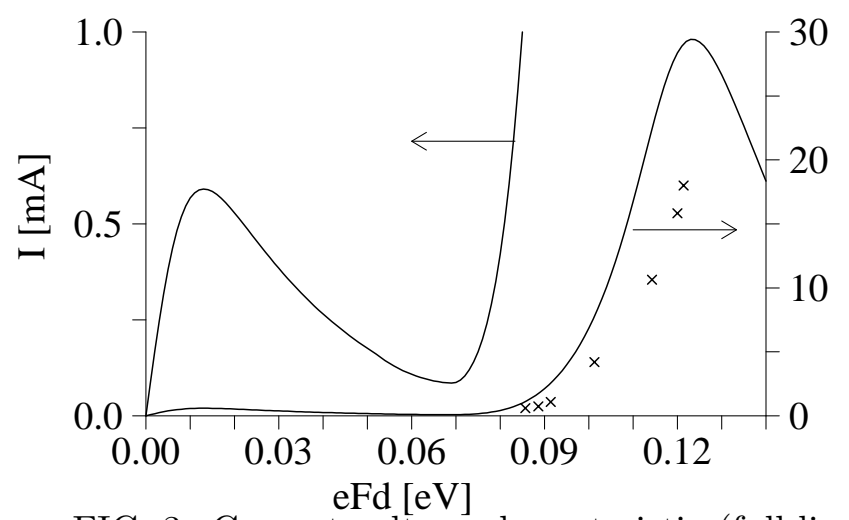

FIG. 3. Current voltage characteristic (full line) for the parameter used in Ref. [17] except for $b=10.5 \mathrm{~nm}$. The data points (crosses) are taken from Fig. 1 of 17]. Note that two different scales at the current axis are used here for the same curve. 


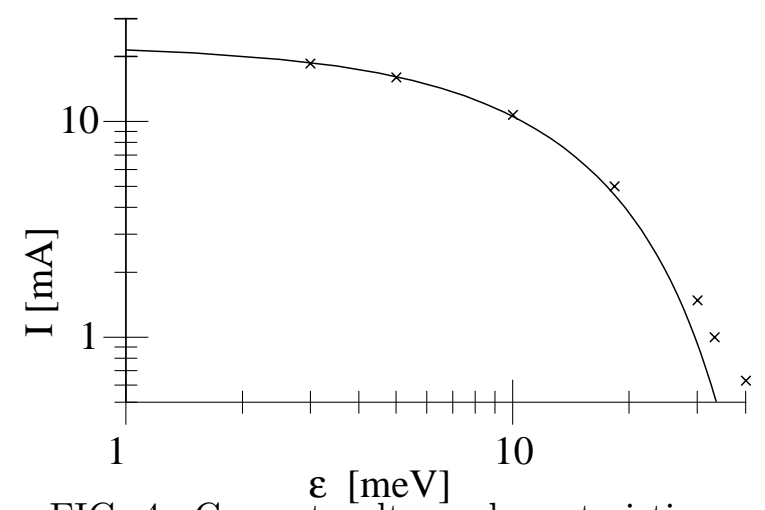

FIG. 4. Current voltage characteristic near the second resonance. The crosses mark experimental data taken from Ref. [17.

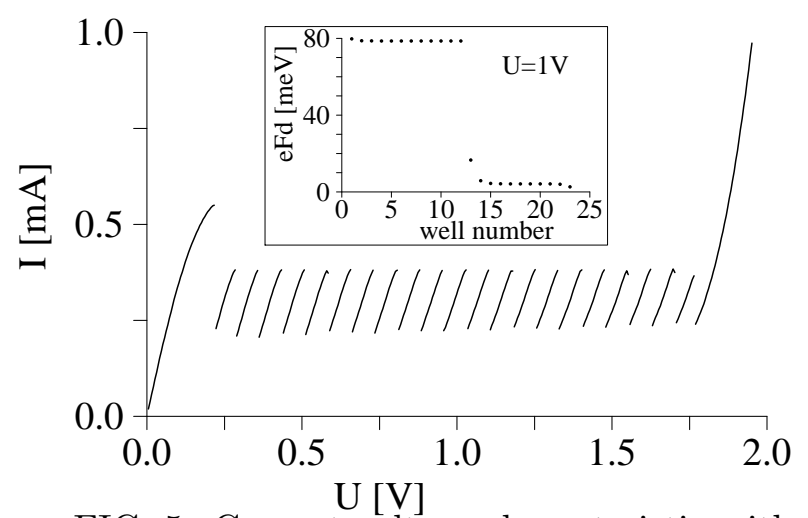

FIG. 5. Current voltage characteristic with domain formation for 23 wells for sweep-up of the voltage. The field distribution for $U=1 \mathrm{~V}$ is shown in the inset where the injecting contact is located at well number 0 . 


\section{TABLES}

\begin{tabular}{|ll|}
\hline \hline$E^{a}$ & $=41.4 \mathrm{meV}$ \\
$E^{b}$ & $=159.3 \mathrm{meV}$ \\
$T_{1}^{a}$ & $=-0.00539 \mathrm{meV}(-0.0152 \mathrm{meV})$ \\
$R_{1}^{b a}$ & $=1.61 \cdot 10^{-4} d\left(4.36 \cdot 10^{-4} d\right)$ \\
$\Gamma_{p h}^{b}$ & $=0.38 \mathrm{meV}$ \\
\hline \hline
\end{tabular}

TABLE I. Calculated parameters for the GaAs- $\mathrm{Al}_{0.3} \mathrm{Ga}_{0.7} \mathrm{As}$ superlattices with well width 8 $\mathrm{nm}$ and barrier width $12 \mathrm{~nm}$. In brackets we have given the respective values for $b=10.5 \mathrm{~nm}$. 